\title{
Molecular Tracking of Coagulase-Negative Staphylococcal Isolates From Catheter-Related Infections
}

\author{
M.A. DOMINGUEZ,' J. LiÑARES,' A. PULIDO,' J.L. PEREZ,' and H. DE LENCASTRE²
}

\begin{abstract}
Three molecular typing methods (pulsed-field electrophoresis, localization of the mecA gene, and probing the vicinity of $\mathrm{mec}$ ) have been used for the characterization of $\mathbf{4 0}$ catheter-related isolates of coagulase-negative staphylococci (CNS) in 14 patients admitted to the same hospital. The 40 isolates yielded 14 different SmaI banding patterns and corresponding unique localizations of $m e c A$, each associated with a unique ClaI $m e c A$ polymorph. In 6 of the 14 patients the contaminated skin at the catheter entry site was the source of 4 local infections and 2 cases of bacteremia. A contaminated hub was the origin of 2 local infections and 4 cases of bacteremia in 6 more patients. The remaining 2 patients had positive cultures from both skin and catheter hub. In each bacteremic patient, the CNS recovered from catheter-related sites (tip, skin, and/or hub) and the CNS recovered from blood were identical, but each of these matching isolates was unique to the particular patient, indicating a low rate of cross-infection from patient to patient. Although classical methods for typing CNS (e.g., biotype and antibiotype) are readily available for most hospital laboratories, they have limitations concerning reproducibility and discriminatory power. Molecular epidemiologic techniques can provide powerful support to traditional techniques in determining the etiologic role of CNS in the disease process.
\end{abstract}

\section{INTRODUCTION}

$\mathbf{T}$ HE MOST COMMON PATHOGENS associated with catheter-related infections (CRIs) are coagulase-negative staphylococci (CNS), specifically Staphylococcus epidermidis. The increase in the use of indwelling devices, along with changes in the patient population, hospital environment, medical practice, and antibiotic policy, have turned these microorganisms into the leading cause of hospital-acquired bacteremia. ${ }^{14,26,30}$

The pathogenesis of infections associated with intravascular catheters is closely related to the cutaneous surface flora. Therefore CNS are the most common etiologic agents related to catheter infections. ${ }^{14,26}$ The two leading routes of catheter contamination are invasion of the catheter external surface by microorganisms present at the insertion site ${ }^{14.24}$ and inner migration of bacteria to the catheter tip through the lumen from a contaminated hub. ${ }^{19,27,30}$ Exogenous microorganisms can be inoculated into the catheter wound or into the hub by contaminated hands of hospital personnel or contaminated antiseptics. ${ }^{14}$
Endogenous microorganisms from other body sites such as the respiratory and gastrointestinal tracts can colonize these areas as well. Hematogenous seeding from a septic distant focus and contamination of the infusate can cause CRI as well. ${ }^{14}$ But none of these routes rivals the importance of the patient's own cutaneous flora contaminating by itself the catheter insertion site or even being transferred to the hub after contacting the patient's skin. ${ }^{14}$

CNS also serve as reservoirs for antibiotic resistance genes ${ }^{3}$ that may be transferred to other gram-positive organisms. This could be the case of the mecA gene. The structural gene mecA, the central genetic determinant of methicillin resistance, has been cloned ${ }^{23,32}$ and found essentially identical in all species of staphylococci, both coagulase-positive and coagulase-negative, that have the characteristic methicillin-resistance phenotype. ${ }^{2,28,29.32}$ The variability associated with the $m e c A$ region has been used as a valuable typing tool among methicillin-resistant Staphylococcus aureus. ${ }^{11,12}$

Efforts to identify the source of CRIs and modes of their

This work was partially presented at the 7th European Congress of Clinical Microbiology and Infectious Diseases, March 1995, Vienna, Austria, (abstract no. 1560).

'Microbiology Department, Hospital de Bellvitge-Prínceps d'Espanya, Barcelona, Spain.

${ }^{2}$ The Rockefeller University, New York, New York 10021, and Instituto de Tecnologia Quimica e Biologica (ITQB), Oeiras, Portugal. 
transmission within the hospital have been hampered by the lack of sufficiently discriminatory typing systems that could distinguish between strains colonizing skin and those causing invasive disease. ${ }^{17,26}$ Traditional epidemiologic analysis needs to be substantiated by more sophisticated molecular techniques, ${ }^{10,30}$ which have been reported as extremely useful in the global evaluation of hospital reservoir of $\mathrm{CNS}^{9,13,15,17,33}$ and in the particular case of CRIs. ${ }^{30}$

The present study evaluates the use of 3 molecular typing methods to characterize $40 \mathrm{CNS}$ isolates associated with infected central venous catheters in 14 patients. Special emphasis is placed on the use of an $S$. aureus mecA gene probe in the intraspecific typing of methicillin-resistant CNS.

\section{MATERIALS AND METHODS}

\section{Bacterial isolates}

Forty catheter-related CNS isolates were collected from 14 inpatients admitted in a 1000-bed hospital in Barcelona, Spain. Seven patients were admitted in a general intensive care unit (cases 1 to 7), and 7 were from a general surgery unit (cases 8 to 14). All patients had central venous, catheters; patients 8 to 14 were receiving total parenteral nutrition. Catheters were removed when suspected of involvement with infection. The signs considered for CRI were any of the following: unexplained fever, localized inflammation, and/or pain on the vascular line and/or on the skin at the catheter entry site. The catheter intravascular segment $(3-4 \mathrm{~cm})$ was sent for microbiologic analysis. Swabs from the hub and skin surrounding the catheter insertion site, taken before catheter withdrawal, were also cultured. Blood cultures, obtained by a distant vein puncture, were performed as well.

\section{Microbiological methods}

Catheter cultures were performed by the semiquantitative (SQ) method $^{22}$ and by the modified quantitative (MQ) method described by Liñares et al. ${ }^{19}$ In the SQ method the catheter tip was transferred to the surface of a blood agar plate and rolled back and forth across the agar surface. After this, the MQ method was carried out by flushing the catheter lumen with 1 $\mathrm{ml}$ tripticase soy broth (Becton-Dickinson Microbiology Systems, Cockeysville, MD), which was then diluted 10-fold, and $0.1 \mathrm{ml}$ of each dilution was plated on blood agar. Colonies were counted after $48 \mathrm{~h}$ of incubation. When 15 or more colonyforming units (CFU) grew on the SQ culture, the catheter was considered infected; if less than 15 grew, the catheter was considered colonized. For the MQ method, more than $10^{3} \mathrm{CFU} / \mathrm{ml}$ was necessary to consider the catheter infected.

CNS recovered from the samples were identified to the species level by an automatized system, Microscan (DADE International, West Sacramento, CA). Antibiotic susceptibility was determined by the disk diffusion and the microdilution methods, according to National Committee for Clinical Laboratory Standards criteria. ${ }^{25}$ The following antibiotics were tested: penicillin, oxacillin, cephalothin, vancomycin, erythromycin, clindamycin, gentamicin, trimethoprim-sulfamethoxazole, fucsidic acid, and ciprofloxacin.

\section{Molecular analysis}

The purification of whole genomic DNA was done according to methods established for $S$. aureus. ${ }^{11}$ Three molecular chromosomal typing methods were employed: (1) pulsed-field gel electrophoresis (PFGE) after DNA restriction with Smal and separation of fragments in a contour-clamped homogeneous electric field (CHEF-DRII) apparatus (Bio-Rad, Richmond, $\mathrm{CA}$ ), with running conditions of $200 \mathrm{~V}$ and pulses ranging from 1 to $30 \mathrm{sec}$ during $23 \mathrm{~h}$ at $14^{\circ} \mathrm{C}$; (2) Smal fragments separated by PFGE were hybridized with the $m e c A$-specific DNA probe ${ }^{11}$ for the localization of the gene on the chromosomal fragments; and (3) digestion of DNA with ClaI followed by hybridization with a $m e c A$-specific DNA probe. DNA fragments were transferred to Hybond-N+ (Amersham, Buckinghamshire, UK) nylon membranes ${ }^{11}$ and hybridized with a nonradioactively labeled probe (ECL, Amersham).

\section{Analysis and interpretation of data}

DNA in PFGE was visualized and photographed in ultraviolet light after the gels were stained with ethidium bromide. PFGE patterns with more than 3-band differences were assigned to distinct clonal types. ${ }^{31}$ Original autoradiograms following hybridization with the $m e c A$ probe were analyzed. Differences in a single band were considered as different mecA polymorphisms. The discriminatory power (DP) percentage in typing CNS was calculated as follows: [number of types/total number of strains] $\times 100$ for a given typing technique. ${ }^{20}$

\section{RESULTS}

\section{Microbiological results}

The 40 catheter-related CNS and their sites of isolation from 14 patients are shown in Table 1. S. epidermidis was recovered from 12 of the 14 patients, and the total number of isolates of $S$. epidermidis was 33. Staphylococcus auricularis (2 isolates) was recovered from patient 5 and Staphylococcus saprophyticus (3 isolates) from patient 11. Staphylococcus haemolyticus was recovered from mixed infections of patient 6 . All 14 catheter tips were found infected by the SQ culture ( $>15 \mathrm{CFU})$ and/or the MQ culture ( $>10^{3} \mathrm{CFU} / \mathrm{ml}$ ). Of the initial CRI in 14 patients, 6 patients had catheter insertion site (skin) cultures that were positive for CNS (cases 2, 3, 5 to 7, and 9); 6 had hub cultures that were positive (cases 1 and 10 to 14); and the remaining 2 patients (cases 4 and 8 ) yielded CNS from both skin and hub cultures. Two patients (cases 6 and 7) had the tip infected with 2 different CNS, both of which were also recovered from the skin in case 6 . Seven patients had catheter-related bacteremia, and the isolate recovered from blood was added to the study. Thus there were 7 cases of catheter-related bacteremia (4 likely originated from the contaminated hub, 2 from the skin insertion site, and 1 with a combined origin) and 7 local catheter infections that did not progress to bacteremia, presumably because the contaminated devices were removed in time.

\section{Phenotypic traits of CNS-related strains}

All CNS were resistant to oxacillin and at least 3 additional antimicrobials. All isolates retained susceptibility to van- 
Table 1. CNS Clinical Sources and Clonal Types

\begin{tabular}{|c|c|c|c|c|c|c|}
\hline $\begin{array}{l}\text { Case \# } \\
\text { (patient) }\end{array}$ & Source & Isolate & $\begin{array}{c}\text { PFGE } \\
\text { pattern } \\
\text { (mecA size) }\end{array}$ & $\begin{array}{c}\text { ClaI } \\
\text { polymorphism }\end{array}$ & $\begin{array}{c}\text { Resistance } \\
\text { profile }^{\mathrm{c}}\end{array}$ & Biotype \\
\hline \multirow[t]{4}{*}{1} & Hub & S. epidermidis & $\mathrm{A}(48.5 \mathrm{~kb})$ & 1 & PenOxErClGenCip & 307164 \\
\hline & Skin & $\mathrm{NC}^{\mathrm{a}}$ & & & & \\
\hline & Tip & S. epidermidis & A $(48.5 \mathrm{~kb})$ & 1 & PenOxErClGenCip & 307164 \\
\hline & Blood & $\mathrm{NC}$ & & & & \\
\hline \multirow[t]{4}{*}{2} & Hub & $\mathrm{NC}$ & & & & \\
\hline & Skin & S. epidermidis & B (90.0 kb) & 2 & PenOxGen & 307120 \\
\hline & Tip & S. epidermidis & B $(90.0 \mathrm{~kb})$ & 2 & PenOxGen & 307120 \\
\hline & Blood & S. epidermidis & B $(90.0 \mathrm{~kb})$ & 2 & PenOxGen & 307120 \\
\hline \multirow[t]{4}{*}{3} & Hub & $\mathrm{NC}$ & & & & \\
\hline & Skin & S. epidermidis & $\mathrm{C}(145.5 \mathrm{~kb})$ & 3 & PenOxErClGen & 307164 \\
\hline & Tip & S. epidermidis & $\mathrm{C}(145.5 \mathrm{~kb})$ & 3 & PenOxErClGen & 307164 \\
\hline & Blood & $\mathrm{NC}$ & & & & \\
\hline \multirow[t]{4}{*}{4} & Hub & S. epidermidis & D $(97.0 \mathrm{~kb})$ & 4 & PenOxGenCip & 307064 \\
\hline & Skin & S. epidermidis & $\mathrm{D}(97.0 \mathrm{~kb})$ & 4 & PenOxGenCip & 307064 \\
\hline & Tip & S. epidermidis & $\mathrm{D}(97.0 \mathrm{~kb})$ & 4 & PenOxGenCip & 307064 \\
\hline & Blood & $\mathrm{NC}$ & & & & \\
\hline \multirow[t]{4}{*}{5} & Hub & $\mathrm{NC}$ & $\mathrm{G}(<48.5 \mathrm{~kb})$ & 7 & PenOx & 302300 \\
\hline & Skin & S. auricularis & $\mathrm{G}(<48.5 \mathrm{~kb})$ & 7 & PenOx & 302300 \\
\hline & Tip & S. auricularis & & & & \\
\hline & Blood & $\mathrm{NC}$ & & & & \\
\hline \multirow[t]{6}{*}{6} & Hub & $\mathrm{NC}$ & & & & \\
\hline & Skin & S. epidermidis & $\mathrm{E}(291.0 \mathrm{~kb})$ & 5 & PenOx & 307120 \\
\hline & & S. haemolyticus & $\mathrm{H}(170.0 \mathrm{~kb})$ & 8 & PenOxErClGen & 302306 \\
\hline & Tip & S. epidermidis & $\mathrm{E}(291.0 \mathrm{~kb})$ & 5 & PenOx & 307120 \\
\hline & & S. haemolyticus & $\mathrm{H}(170.0 \mathrm{~kb})$ & 8 & PenOxErClGen & 302306 \\
\hline & Blood & $\mathrm{NC}$ & & & & \\
\hline \multirow[t]{5}{*}{7} & Hub & $\mathrm{NC}$ & & & & \\
\hline & Skin & S. epidermidis & $\mathrm{F}(200.0 \mathrm{~kb})$ & 6 & PenOxErClGen & 307064 \\
\hline & Tip & S. epidermidis (1) & $\mathrm{A}(48.5 \mathrm{~kb})$ & 1 & PenOxErCiGenCip & 307164 \\
\hline & & S. epidermidis (2) & $\mathrm{F}(200.0 \mathrm{~kb})$ & 6 & PenOxErClGen & 307064 \\
\hline & Blood & $\mathrm{NC}$ & & & & \\
\hline \multirow[t]{4}{*}{8} & Hub & S. epidermidis & I (193 kb) & 9 & PenOxErCl & 306164 \\
\hline & Skin & S. epidermidis & I (193 kb) & 9 & PenOxErCl & 306164 \\
\hline & Tip & S. epidermidis & I (193 kb) & 9 & PenOxErCl & 306164 \\
\hline & Blood & S. epidermidis & I (193 kb) & 9 & PenOxErCl & 306164 \\
\hline \multirow[t]{4}{*}{9} & Hub & $\mathrm{NC}$ & & & & \\
\hline & Skin & S. epidermidis & $\mathrm{J}(400 \mathrm{~kb})$ & 10 & PenOxErCl & 303144 \\
\hline & Tip & S. epidermidis & $\mathrm{J}(400 \mathrm{~kb})$ & 10 & PenOxErCl & 303144 \\
\hline & Blood & S. epidermidis & $\mathrm{J}(400 \mathrm{~kb})$ & 10 & PenOxErCl & 303144 \\
\hline \multirow[t]{4}{*}{10} & Hub & S. epidermidis & $\mathrm{K}(150.5 \mathrm{~kb})$ & 11 & PenOxErCl & 307044 \\
\hline & Skin & $\mathrm{NC}$ & & & & \\
\hline & Tip & S. epidermidis & $\mathrm{K}(150.5 \mathrm{~kb})$ & 11 & PenOxErCl & 307044 \\
\hline & Blood & $\mathrm{NC}$ & & & & \\
\hline \multirow[t]{4}{*}{11} & Hub & S. saprophyticus & $\mathrm{L}$ & $\mathrm{NH}^{\mathrm{b}}$ & PenOxErClTS & 246022 \\
\hline & Skin & $\mathrm{NC}$ & & & & \\
\hline & Tip & S. saprophyticus & $\mathrm{L}$ & NH & PenOxErClTS & 246022 \\
\hline & Blood & S. saprophyticus & $\mathbf{L}$ & NH & PenOxErClTS & 246022 \\
\hline
\end{tabular}


TABLE 1. (continued)

\begin{tabular}{|c|c|c|c|c|c|c|}
\hline $\begin{array}{l}\text { Case \# } \\
\text { (patient) }\end{array}$ & Source & Isolate & $\begin{array}{c}P F G E \\
\text { pattern } \\
\text { (mecA size) }\end{array}$ & $\begin{array}{c}\text { ClaI } \\
\text { polymorphism }\end{array}$ & $\begin{array}{c}\text { Resistance } \\
\text { profile }^{\mathrm{c}}\end{array}$ & Biotype \\
\hline \multirow[t]{3}{*}{12} & $\begin{array}{l}\text { Hub } \\
\text { Skin }\end{array}$ & $\begin{array}{l}\text { S. epidermidis } \\
\text { NC }\end{array}$ & I (193 kb) & 9 & PenOx & 306164 \\
\hline & Tip & S. epidermidis & I (193 kb) & 9 & PenOx & 306164 \\
\hline & Blood & S. epidermidis & I (193 kb) & 9 & PenOx & 306164 \\
\hline \multirow[t]{3}{*}{13} & Hub & S. epidermidis & M (400 kb) & 12 & PenOxErClTS & 307164 \\
\hline & $\begin{array}{l}\text { SkIn } \\
\text { Tip }\end{array}$ & S. epidermidis & M (400 kb) & 12 & PenOxErClTS & 307164 \\
\hline & Blood & S. epidermidis & M $(400 \mathrm{~kb})$ & 12 & PenOxErClTS & 307164 \\
\hline \multirow[t]{3}{*}{14} & Hub & S. epidermidis & $\mathrm{N}(138.5 \mathrm{~kb})$ & 13 & PenOxErClGenTS & 307164 \\
\hline & Skin & $\mathrm{NC}$ & & & & \\
\hline & Tip & $\begin{array}{l}\text { S. epidermials } \\
\text { S. epidermidis }\end{array}$ & $\begin{array}{l}\mathrm{N}(138.5 \mathrm{~kb}) \\
\mathrm{N}(138.5 \mathrm{~kb})\end{array}$ & $\begin{array}{l}13 \\
13\end{array}$ & $\begin{array}{l}\text { PenOxErClGenTS } \\
\text { PenOxErClGenTS }\end{array}$ & 307164 \\
\hline
\end{tabular}

${ }^{a} \mathrm{NC}$, negative culture.

bNH, not homologous with mecA probe.

'Resistance profile, includes resistance to penicillin, -Pen-; oxacillin, -Ox; erythromycin, -Er-; clindamycin, -Cl-; gentamicin,

-Gen-; trimethroprim-sulfamethoxazol, -TS-; and ciprofloxacin, -Cip-.

comycin. Susceptibility to other antimicrobial agents such as aminoglycosides, fluoroquinolones, macrolides, or trimethoprim-sulfamethoxazole was variable. Altogether, 8 distinct resistance profiles were found (Table 1), which were used as epidemiologic markers as well (DP, 20\%).

Biotyping has resolved the 33 catheter-related isolates of $S$. epidermidis into 6 biotypes (Table 1). Three species other than $S$. epidermidis ( $S$. auricularis, S. haemolyticus, and $S$. saprophyticus) were assigned to three additional biotypes. Thus biotyping had an assigned DP of $22.5 \%$.

The CNS recovered from all different catheter-related sites showed the same phenotypic traits (biotype and antibiotype) in each patient (Table 1).

\section{Molecular traits of CNS-related strains}

DNA fingerprinting of the 33 isolates of $S$. epidermidis (isolated from 12 patients) yielded the following results (Table 1): PFGE after Smal digestion of chromosomal DNA resolved them into 11 different banding patterns (A to $\mathrm{F}, \mathrm{I}$ to $\mathrm{K}, \mathrm{M}$ and $\mathrm{N}$ ), each one with a characteristic location of the mecA gene and each carrying a unique mecA polymorph as well. For instance, the two CNS isolates in case 1 showed a unique PFGE pattern (Fig. 1, A); the mecA gene probe hybridized with a 48-kb SmaI fragment (Fig. 1, B); and mecA polymorph 1 was present (Fig. 2 , A). In contrast, the $3 \mathrm{CNS}$ isolates of case 2 shared a common PFGE pattern B (Fig. 1, A) and a ClaI mecA polymorph 2 (Fig. 2, A). In this group of isolates the mecA probe hybridized with a 90-kb SmaI fragment (Fig. 1, B). Similarly, the remaining $28 \mathrm{~S}$. epidermidis isolates each had unique PFGE patterns, carried mecA polymorphs, and showed mecA-hybridizing fragments of unique molecular size.

The 2 pairs of $S$. auricularis and $S$. haemolyticus isolates (cases 5 and 6) belonged to 2 additional and unique PFGE types named $\mathrm{G}$ and $\mathrm{H}$, each with its characteristic mecA location and carrying 2 corresponding, unique mecA polymorphs (polymorphs 7 and 8). Three $S$. saprophyticus isolates from case 11 also had a single PFGE pattern but did not show any homology with the mecA fragment used as probe; thus no mecA hybridizing pattern was found. All together, of $40 \mathrm{CNS}$ isolates, PFGE yielded 14 different patterns (DP, 35\%) and ClaI mecA hybridization, 13 types (DP, 32.5\%).

As shown in Table 1, in every single patient a unique and identical CNS clone (as defined by the molecular, biochemical, and antibiotic resistance profiles) could be recovered from all different catheter-related sites. Furthermore, in the 7 bacteremic patients bacteria recovered from blood cultures were identical to the bacteria isolated at the catheter sites of each particular patient. We conclude that extraluminal contamination of the catheter's external surface from the skin at the catheter entry site has been the most likely origin of 4 local infections (cases 3, 5, 6, and 7) and 2 cases of bacteremia (cases 2 and 9). The inner catheter surface was most likely contaminated from a contaminated hub in 6 patients and was the origin of 2 local infections (cases 1 and 10) and 4 bacteremias (cases $11,12,13$, and 14). In cases 4 and 8 , positive cultures were obtained from both skin and hub. Presumably, in these cases hubs were contaminated by inoculating the patient's own skin flora in the subsequent manipulation of the central line.

\section{DISCUSSION}

The pathogenesis of central catheter infections and the role of CNS have been properly established since the early $1980 \mathrm{~s}^{19,21}$ Since then, every scientific review of the field remarks the need for more reliable tools to conclusively prove the relatedness of the isolated strains. ${ }^{14,26,30}$ Methods that still are recommended for typing CNS are the biochemical profile and the antimicrobial sus- 
A
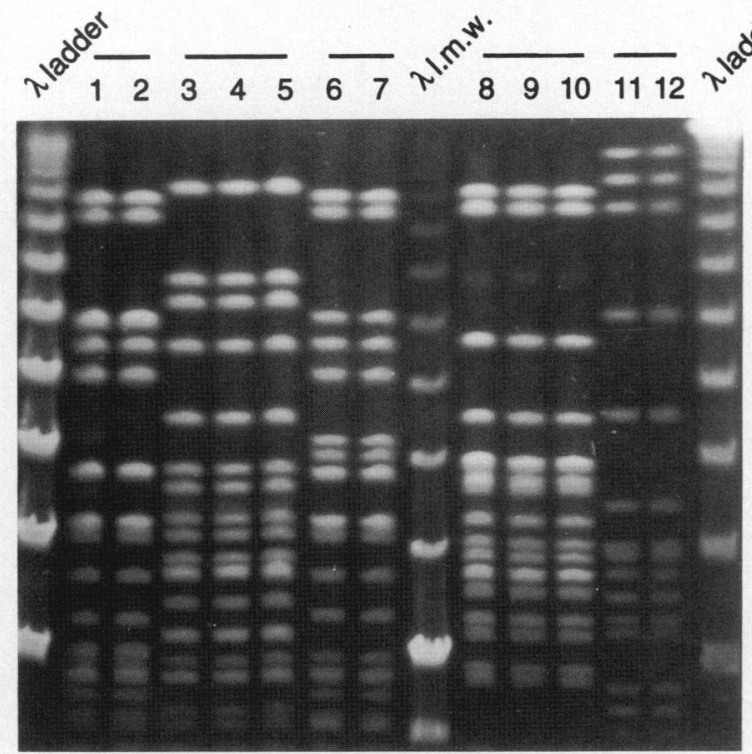

B

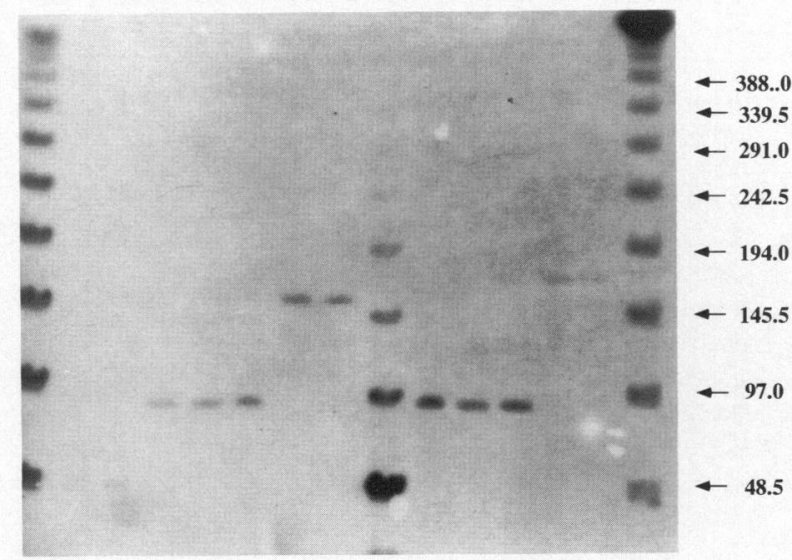

FIG. 1. Chromosomal backgrounds and localization of $m e c A$ in isolates of coagulase-negative streptococci (CNS). (A) Chromosomal DNAs of 12 CNS isolates from 5 patients were restricted with SmaI. Five unique patterns were detected by pulsed-field gel electrophoresis (PFGE). Among the 10 Staphylococcus epidermidis isolates, patterns were shared by isolates 1 and 2 (pattern A), 3-5 (pattern B), 6 and 7 (pattern C), and 8-10 (pattern D). Lanes 11 and 12 contain Staphylococcus haemolyticus pattern H. (B) The same gel shown was hybridized with the $m e c A$ DNA probe to localize the gene to various $S m a I$ fragments. $\lambda$ ladder and $\lambda$ l.m.w. (low molecular weight) are lambda ladder PFG markers (New England BioLabs, Beverly, MA).

ceptibility pattern. ${ }^{7}$ Both biotype and antibiotype are readily available on a timely and affordable basis for most hospital laboratories. However, they have recognized limitations. They do not always give reproducible results ${ }^{10}$; unique patterns may result from local conditions, such as antibiotic pressure, and may not be stable over time or geographic distance. Appearance of unique biochemical or susceptibility patterns may serve as a preliminary marker that will need to be confirmed by complementary techniques. In our study biotype or antibiotype was shown to be use- ful in analyzing CNS from distinct catheter-related sites when a single patient was considered. Considered separately, neither biotype nor antibiotype was adequate in tracing routes of CNS transmission among different patients (i.e., isolates with the same biotype or antibiotype belonged to different clonal types, as confirmed by molecular methods). However, if both phenotypic typing techniques were considered together, their DP value rose to $37 \%$, a percentage even higher than that obtained with the molecular tools applied here (35\%). It should be mentioned at this point that the automated system used in our clinical laboratory for CNS speciation contained 26 biochemical tests and 21 antibiotics.

Many sophisticated and molecular techniques have been applied recently for studying the epidemiology of CNS infections. Some examples are multilocus enzyme electrophoresis, ${ }^{30}$ plasmid DNA analysis, ${ }^{4,10,24}$ restriction endonuclease fingerprinting of the chromosomal DNA ${ }^{4}$ and hybridization with specific nucleic acid probes, ${ }^{3,59}$ PFGE after genomic DNA macrorestriction, ${ }^{8,10}$ and polymerase chain reaction-based typing techniques. ${ }^{6}$

In the study described here, we used 3 molecular typing tools that have already demonstrated good discriminatory power in the analyses of methicillin-resistant $S$. aureus (MRSA) hospital outbreaks. ${ }^{5,11}$ Two of these typing tools were based on determining mecA polymorphism after digestion of chromosomal DNA with SmaI or ClaI. All but 1 of the CNS isolates studied here were nosocomially acquired, and all of them were multidrug-resistant

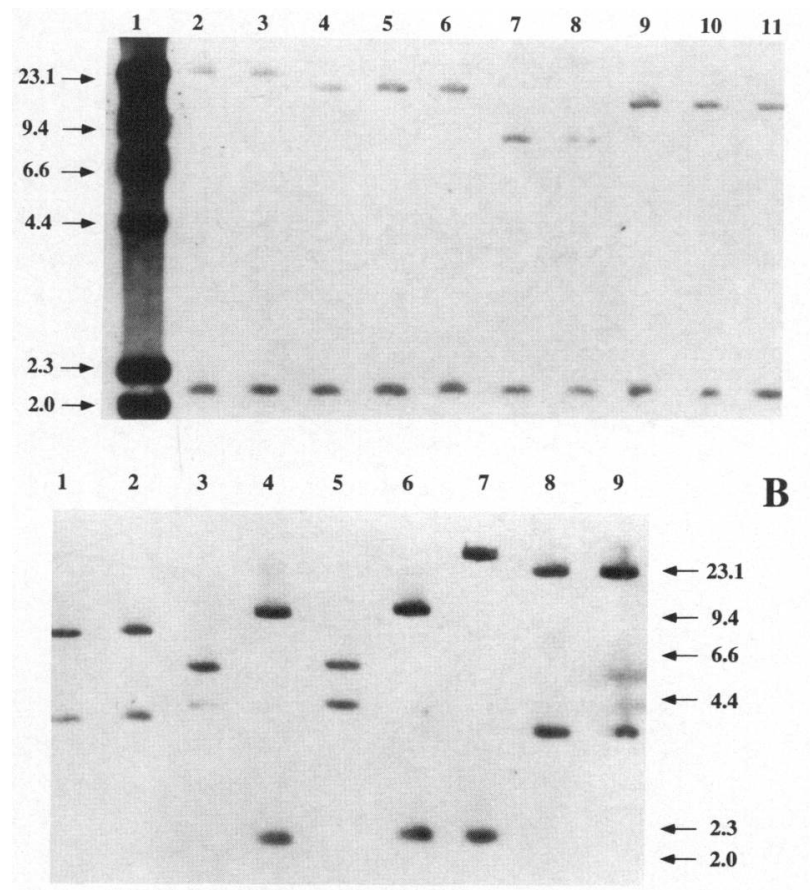

FIG. 2. Chromosomal DNAs from CNS were restricted with $C l a \mathrm{I}$ and hybridized with the $m e c A$ probe; $m e c A$ polymorphs were assigned. (A) The same isolates of $S$. epidermidis as in Figure 1 are shown ( $S$. haemolyticus is not shown). Isolates from cases 1 to 4 showed polymorphs $1,2,3$, and 4, respectively. (B) Case 5: Staphylococcus auricularis, polymorphism type 7. Case 6: $S$. haemolyticus (lanes 3 and 5), polymorphism type 8; and $S$. epidermidis (lanes 4 and 6), polymorphism type 5. Case 7: S. epidermidis (lane 7), type 1; and S. epidermidis (lanes 8 and 9), type 6. 
and methicillin-resistant, allowing the use of the mecA gene as a molecular marker. This would remain the case for the majority of nosocomial CNS infections, since in our nosocomial environment, about $70 \%$ of CNS are oxacillin resistant. A similar figure was reported by the National Nosocomial Infection Survey for CNS isolates in the United States, in 1989, with the most frequent coagulase-negative species being $S$. epidermidis. ${ }^{1,15}$

Our findings indicate that the DP of the mecA probe may be higher than that reported for ribotyping ${ }^{16}$ among CNS. Also, it showed higher DP among CNS than among MRSA isolates. Only relative few mecA polymorphs have been identified among several hundred MRSA strains collected from a wide variety of geographic sources, ${ }^{18}$ and the mecA hybridization patterns of hospital outbreaks of MRSA disease are usually associated with a dominant MRSA clone carrying a single mecA polymorph. ${ }^{11,12}$ This is in sharp contrast to the observations described in this communication: the CNS isolates collected at a single hospital site within a relatively short period showed a wide range of variation in the vicinity and localization of the mec gene, and also in the general chromosomal backgrounds of the isolates. This fact has allowed an unambiguous determination of the origins and transmission routes of the CNS isolates involved with the 14 CRIs described here.

Previous studies have demonstrated that some CNS can become endemic over long periods and can be transmitted among patients by hospital personnel. ${ }^{9,15}$ In our particular set of CNS infections, there were only 2 cases of potential cross-infection: clone A::1 (PFGE::mecA polymorph) from case 1 (isolated from skin and catheter tip), which was also found in case 7 (from catheter tip) 2 months later; and clone I::9, which was found in case 8 , and 3 months later in case 12 .

The introduction of methods such as those used in this study should generally improve the management of nosocomial infections by CNS strains. CNS infections are notoriously difficult to treat, both because of the patient population affected (long-term hospitalized patients, often with severe underlying disease) and because of the multidrug resistance expressed by these bacteria. CNS are also the most common contaminants in the hospital environment, and molecular techniques offer the high discriminatory power essential for distinguishing contaminants from the true etiologic agents of disease.

\section{ACKNOWLEDGMENTS}

M.D. Dominguez was supported by grant no. 93/5178. This work was partially supported by grant no. 92/1127. Both grants are from Fondo de Investigacion Sanitaria, National Institute of Health, Madrid, Spain. Partial support for this work was provided by the Centro de Epidemiologia Molecular (CEM), Oeiras, Portugal, and from the Rockefeller University.

\section{REFERENCES}

1. Archer, G.L., and M. Climo. 1994. Antimicrobial susceptibility of coagulase-negative staphylococci. Antimicrob. Agents Chemother. 38:2231-2237.

2. Archer, G.L., and D.M. Niemeyer. 1994. Origin and evolution of DNA associated with resistance to methicillin in staphylococci. Trends Microbiol. 2:343-347.

3. Archer, G.L., and J.L. Johnston. 1983. Self-transmissible plas- mids in staphylococci that encode resistance to aminoglycosides. Antimicrob. Agents Chemother. 23:151-160.

4. Bialkowska-Hobrazanska, H., D. Jaskot, and O. Hammerberg. 1990. Evaluation of restriction endonuclease fingerprinting of chromosomal DNA and plasmid profile analysis for characterization of multiresistant coagulase-negative staphylococci in bacteremic neonates. J. Clin. Microbiol. 28:269-275.

5. Bialkowska-Kobrazanska H., V. Harry, and O. Hammerberg. 1990. Typing of coagulase-negative staphylococci by southern hybridization of chromosomal DNA fingerprints using a ribosomal RNA probe. Eur. J. Clin. Microbiol. Infect. Dis. 9:588-594.

6. Bingen, E., M.C. Carc, C. Brahimi, E. Vilmer, and F. Beaufils. 1995. Randomly amplified polymorphic DNA analysis provides rapid differentiation of methicillin-resistant coagulase-negative staphylococcus bacteremia isolates in pediatric hospital. J. Clin. Microbiol. 33:1657-1659.

7. Birnbaum, D., M. Kelly, and A.W. Chow. 1991. Epidemiologic typing systems for coagulase-negative staphylococci. Infect. Control Hosp. Epidemiol. 2:319-326.

8. Breen, J.D., and A.W. Karchmer. 1994. Usefulness of pulsedfield gel electrophoresis in confirming endocarditis due to Staphylococcus lugdunensis. Clin. Infect. Dis. 19:985-986.

9. Degener, J.E., M.E.O.C. Hecn, W.J. van Leeuwen, C. Heemskerk, A. Crielaard, P. Joosten, and P. Caesar. 1994. Nosocomial infection by Staphylococcus haemolyticus and typing methods for epidemiological study. J. Clin. Microbiol. 32:2260-2265.

10. Deighton, M., S. Pearson, J. Capstick, D. Spelman, and R. Borland. 1992. Phenotypic variation of Staphylococcus epidermidis isolated from a patient with native valve endocarditis. J. Clin. Microbiol. 30:2385-2390.

11. De Lencastre, H., I. Couto, I. Santos, J. Melo-Cristino, A. Torres-Pereira, and A. Tomasz. 1994. Methicillin-resistant Staphylococcus aureus disease in a Portuguese hospital: Characterization of clonal types by a combination of DNA typing methods. Eur. J. Clin. Microbiol. Infect. Dis. 13:64-73.

12. Dominguez, M.A., H. de Lencastre, J. Liñares, and A. Tomasz. 1994. Spread and maintenance of a dominant methicillin-resistant Staphyloccus aureus (MRSA) clone during an outbreak of MRSA disease in a Spanish hospital. J. Clin. Microbiol. 32:2081-2087.

13. Goering, R.V., and T.D. Duensing. 1990. Rapid field inversion gel electrophoresis in combination with an rRNA gene probe in the epidemiological evaluation of staphylococci. J. Clin. Microbiol. 28:426-429.

14. Goldmann, D.A., and G. Pier. 1993. Pathogenesis of infections related to intravascular catheterization. Clin. Microbiol. Rev. 6:176-192.

15. Huebner, J., G.B. Pier, J. Maslow, E. Muller, H. Shiro, M. Parent, A. Kropec, R. Arbeit, and D.A. Goldmann. 1994. Endemic nosocomial transmission of Staphylococcus epidermidis bacteremia isolates in a neonatal intensive care unit over 10 years. J. Infect. Dis. 169:526-531.

16. Izard, N., Hächler, M. Grehn, and F.H. Kayser. 1992. Ribotyping of coagulase-negative staphylococci with special emphasis on intraspecific typing of Staphylococcus epidermidis. J. Clin. Microbiol. 30:817-823.

17. John, J.F., T. Grieshop, L. Atkins, and C.G. Platt. 1993. Widespread colonization of personnel at a veterans affairs medical center by methicillin-resistant, coagulase-negative Staphylococcus. Clin. Inf. Dis. 17:380-388.

18. Kreiswirth, B., J. Kornblum, R.D. Arbeit, W. Eisner, J.N. Maslow, A. McGeer, D.E. Low, and R.P. Novick. 1993. Evidence for a clonal origin of methicillin resistance in Staphylococcus aureus. Science 259:227-230.

19. Liñares, J., A. Sitges-Serra, J. Garau, J.L. Perez, and R. Martin. 1985. Pathogenesis of catheter sepsis: A prospective study with quantitative and semiquantitative cultures of catheter hub and segments. J. Clin. Microbiol. 21:357-360.

20. Ludlam, H.A., W.C. Noble, R.R. Marples, and I. Phillips. 1989. 
The evaluation of a typing scheme for coagulase-negative staphylococci suitable for epidemiological studies. J. Med. Microbiol. 30:161-165.

21. Maki, D.G., and C.J. Alvarado. 1982. The role of clinical microbiology laboratory in diagnosis of infusion related sepsis. Clin. Microbiol. Newsletter 4:89-94.

22. Maki, D.G., Weise, C.E., and H.W. Sarafin. 1977. A semiquantitative culture method for identifying intravenous catheter related infection. N. Engl. J. Med. 296:1305-1309.

23. Matsuhashi, M., M.D. Song, F. Ishino, M. Wachi, M. Doi, M. Inoue, K. Ubukata, N. Yamashita, and M. Konno. 1986. Molecular cloning of the gene of a penicillin-binding protein supposed to cause high resistance to $\beta$-lactam antibiotics in Staphylococcus aureus. J. Bacteriol. 167:975-980.

24. Mermel, L.A., R.D. McCormick, S.R. Springman, and D.G. Maki. 1991. The pathogenesis and epidemiology of catheter-related infection with pulmonary artery Swan-Ganz catheters: A prospective study utilizing molecular subtyping. Am. J. Med. 91(3B):197s-205s.

25. National Committee for Clinical Laboratory Standards. 1990. Performance standards for antimicrobial disk susceptibility test, 4 th ed. Approved standard M2-A4. National Committee for Clinical Laboratory Standards, Villanova, PA.

26. Rupp, M.E., and G. Archer. 1994. Coagulase-negative staphylococci: Pathogens associated with medical progress. Clin. Infect. Dis. 19:231-245.

27. Sitges-Serra, A., P. Puig, J. Liñares, JL. Perez, N. Farrero, E. Jaurrieta, and J. Garau. 1984. Hub colonization as the initial step in an outbreak of catheter-related sepsis due to coagulase-negative staphylococci during parenteral nutrition. J. Parenteral Enteral Nutr. 8:668-672.
28. Suzuki, E., K. Hiramatsu, and T. Yokota. 1992. Survey of methicillin-resistant clinical strains of coagulase-negative staphylococci for mecA gene distribution. Antimicrob. Agents Chemother. 36:429-434.

29. Suzuki, E., K. Kyoko, J.F. Ricardson, and K. Hiramatsu. 1993. Distribution of mec regulator genes in methicillin-resistant Staphylococcus clinical strains. Antimicrob. Agents Chemother. 37:1219-1226.

30. Tan, T.Q., J.M. Musser, R. Shulman, E. Mason, D.H. Mahoney, and S.L. Kaplan. 1994. Molecular epidemiology of coagulasenegative Staphylococcus blood isolates from neonates with persistent bacteremia and children with central venous catheter infections. J. Infect. Dis. 169:1393-1397.

31. Tenover, F.C., R.D. Arbeit, R.V. Goering, P.A. Mickelsen, B.E. Murray, D.H. Persing, and B. Swaminathan. 1995. Interpreting chromosomal DNA restriction patterns produced by pulsed field electrophoresis: Criteria for bacterial strain typing. J. Clin. Microbiol. 33:2233-2239.

32. Tesch, W., A. Strässle, B. Berger-Bächi, D. O'Hara, P. Reynolds, and F.H. Kayser. 1988. Cloning and expression of methicillin resistance from Staphylococcus epidermidis in Staphylococcus carnosus. Antimicrob. Agents Chemother. 32:1494-1499.

33. Wilton, J., K. Jung, I. Vedin, B. Aronsson, and J.I. Flock. 1992. Comparative evaluation of a new molecular method for typing Staphylococcus epidermidis. Eur. J. Clin. MicrobioI. Infect. Dis. 11:515-521.

Address reprint requests to:

H. de Lencastre

The Rockefeller University 1230 York Avenue New York, NY 10021 


\section{This article has been cited by:}

1. SUSAN E. BEEKMANN, DAVID K. HENDERSONInfections Caused by Percutaneous Intravascular Devices 3697-3715. [CrossRef]

2. Ivana Pilipčincová, Mangesh Bhide, Eva Dudriková, Milan Trávniček. 2010. Genotypic Characterization of Coagulasenegative Staphylococci Isolated from Sheep Milk in Slovakia. Acta Veterinaria Brno 79:2, 269-275. [CrossRef]

3. Yhu-Chering Huang, Ying-Hsiang Wang, Lin-Hui Su, Yi-Hong Chou, Rey-In Lien, Tzou-Yien Lin. 2006. Determining the Significance of Coagulase-Negative Staphylococci Identified in Cultures of Paired Blood Specimens from Neonates by Species Identification and Strain Clonality - . Infection Control and Hospital Epidemiology 27:1, 70-73. [CrossRef]

4. M. Miragaia, I. Couto, Dr. H. De Lencastre. 2005. Genetic Diversity among Methicillin-Resistant Staphylococcus epidermidis (MRSE). Microbial Drug Resistance 11:2, 83-93. [Abstract] [Full Text PDF] [Full Text PDF with Links]

5. Silvia F Costa, Marisa H Miceli, Elias J Anaissie. 2004. Mucosa or skin as source of coagulase-negative staphylococcal bacteraemia?. The Lancet Infectious Diseases 4:5, 278-286. [CrossRef]

6. I. Spiliopoulou, I. Santos Sanches, C. Bartzavali, A.M. Ludovice, M. Aires de Sousa, G. Dimitracopoulos, H. de Lencastre. 2003. Application of Molecular Typing Methods to Characterize Nosocomial Coagulase-Negative Staphylococci Collected in a Greek Hospital During a Three-Year Period (1998-2000). Microbial Drug Resistance 9:3, 273-282. [Abstract] [Full Text PDF] [Full Text PDF with Links]

7. E Marques de Mattos. 2003. Isolation of methicillin-resistant coagulase-negative staphylococci from patients undergoing continuous ambulatory peritoneal dialysis (CAPD) and comparison of different molecular techniques for discriminating isolates of Staphylococcus epidermidis. Diagnostic Microbiology and Infectious Disease 45:1, 13-22. [CrossRef]

8. J. Carratala. 2001. Role of antibiotic prophylaxis for the prevention of intravascular catheter-related infection. Clinical Microbiology and Infection 7:4, 83-90. [CrossRef]

9. Massoumeh D. Nahvi, Joseph E. Fitzgibbon, Joseph F. John, Donald T. Dubin. 2001. Sequence Analysis of dru Regions from Methicillin-Resistant Staphylococcus aureus and Coagulase-Negative Staphylococcal Isolates. Microbial Drug Resistance 7:1, 1-12. [Abstract] [Full Text PDF] [Full Text PDF with Links]

10. Soon-Duck Kim, L. Clifford McDonald, William R. Jarvis, Sigrid K. McAllister, Robert Jerris, Loretta A. Carson, J. Michael Miller. 2000. Determining the Significance of Coagulase-Negative Staphylococci Isolated from Blood Cultures at a Community Hospital: A Role for Species and Strain Identification - Infection Control and Hospital Epidemiology 21:3, 213-217. [CrossRef]

11. Jesús Fortún, Enrique Navas. 1999. A critical approach to the pathogenesis, diagnosis, treatment and prevention of catheter-related bloodstream infections and nosocomial endocarditis. Clinical Microbiology and Infection 5, 2s40-2s50. [CrossRef]

12. C STRATTON. 1998. Catheter-associated infections: A necessary evil. Antimicrobics and Infectious Diseases Newsletter 17:7, 49-54. [CrossRef] 